\title{
Multistable Dynamics in a Hopfield Neural Network Under Electromagnetic Radiation and Dual Bias Currents
}

\author{
Qiuzhen Wan ( $\nabla$ wanqiuzhen@sina.com ) \\ Hunan Normal University https://orcid.org/0000-0003-1093-5582
}

\section{Zidie Yan}

Hunan Normal University

Fei Li

Hunan Normal University

Jiong Liu

Hunan Normal University

\section{Simiao Chen}

Hunan Normal University

\section{Research Article}

Keywords: Hopfield neural network (HNN), electromagnetic radiation, dual bias currents, multistability, transient chaos, parallel bifurcation.

Posted Date: December 2nd, 2021

DOI: https://doi.org/10.21203/rs.3.rs-1121450/v1

License: (c) (1) This work is licensed under a Creative Commons Attribution 4.0 International License. Read Full License 


\title{
Multistable dynamics in a Hopfield neural network under electromagnetic radiation and dual bias currents
}

\author{
Qiuzhen Wan *, Zidie Yan, Fei Li, Jiong Liu, Simiao Chen \\ College of Information Science and Engineering, Hunan Normal University, Changsha 410081, P. R. China
}

\begin{abstract}
This paper investigates a Hopfield neural network (HNN) under the simulation of external electromagnetic radiation and dual bias currents, in which the fluctuation of magnetic flux across the neuron membrane is used to emulate the influence of electromagnetic radiation. Utilizing conventional analytical methods, the basic properties of the proposed Hopfield neural network are discussed. Due to the addition of electromagnetic radiation and dual bias currents, the Hopfield neural network shows high sensitivity to system parameters and initial conditions. The proposed Hopfield neural network possesses multistability with periodic attractor, quasi-periodic attractor, chaotic attractor and transient chaotic attractor, and all of the attractors are hidden attractors because there is no equilibrium point in the system. In particular, when the neuron membrane magnetic flux is different, the system can present transient chaos with different chaotic times. More interestingly, with the change of system parameters, the proposed Hopfield neural network can exhibit parallel bifurcation behaviors. Finally, the Multisim simulation and hardware experiment results based on discrete electronic components are conducted to support the numerical ones. These results could give useful information to the study of nonlinear dynamic characteristics of the Hopfield neural network.
\end{abstract}

Keywords: Hopfield neural network (HNN); electromagnetic radiation; dual bias currents; multistability; transient chaos; parallel bifurcation.

*Corresponding author. E-mail address: wanqiuzhen@sina.com (Qiuzhen Wan) 


\section{Introduction}

The brain is composed of a large number of nerve cells or neurons, and each neuron can be regarded as a small processing unit. These neurons are connected to each other in a certain way to form the biological neural network inside the brain. Humans rely on the brain for thinking, associating, memorizing, and reasoning. For a long time, many researchers have been committed to the study of the internal structure and function of the human brain. Nowadays, there is evidence that various complex activities in the brain are carried out through neural network ${ }^{[1-3]}$. Therefore, the researches on neural network are very necessary.

As the fourth basic circuit element, memristor has received great attention since it was firstly proposed by Chua in $1971^{[4]}$. Since the memristor is a passive two-terminal component, its plasticity and non-volatility are closely related to the memory characteristic of neurons ${ }^{[5,6]}$, so the memristor is widely employed in the researches of neural network. For example, Chen et al. ${ }^{[7]}$ adopted an ideal flux-controlled memristor synapse to simulate the induced current generated by the potential difference between two neurons, upon which they found several extraordinary coexisting multi-stable patterns. Tang et al. ${ }^{[8]}$ proposed a non-spike timing-dependent plasticity learning mechanism for memristive neural networks. This work has been achieved better hardware performance, and provided a broader space for the practical application of memristive neural networks. Xiu et al. ${ }^{[9]}$ designed a new memristor model with smooth characteristic curve, and employed it to construct a four-dimensional memristive cellular neural network system. Its chaotic dynamic behaviors can be applied to secure communication. Zhang et al. ${ }^{[10]}$ studied synchronization problem of coupled memristive neural networks, and Cao et al. ${ }^{[11]}$ discussed exponential anti-synchronization problem. Besides, Liu et al. ${ }^{[12]}$ investigated the dynamical robustness and transition of firing modes of multilayer memristive neural networks in detail. All these works show that the memeistor can effectively promote the researches of neural network in different fields. If readers want to know more cases of neural network based on memristor, the review ${ }^{[13]}$ and the references in it are good choices.

In order to better investigate the neural network in the human brain, artificial neural network has been proposed. Artificial neural network abstracts the human brain neural network from the perspective of information processing, and establishes a simple model to simulate the real biological activities in the human brain, among which the Hopfield neural network (HNN) is a typical one ${ }^{[14]}$. It clarifies the relationship between neural network and dynamics, and has a clear correspondence with electronic circuit, which makes HNN easy to understand and can be implemented using integrated circuit. There are many 
factors that can affect the dynamic behaviors of the Hopfield neural network, including coupling weights ${ }^{[15-18]}$, network structure ${ }^{[19,20]}$, neuron activation gradient ${ }^{[21,22]}$, time delay ${ }^{[23,24]}$ etc. In particular, due to the application of various electronic products in the real world, electromagnetic radiation is one of the most important factors that cannot be ignored. Hu et al. ${ }^{[25]}$ firstly studied a small Hopfield neural network with three neurons, which found that periodic motion and chaotic motion appeared intermittently with variations in some system parameters, and this may explain the intermittent convulsions in patients with Epilepsy ${ }^{[26,}$ ${ }^{27]}$. Then, Lin et al. ${ }^{[28]}$ explored the dynamics of different numbers of neurons affected by electromagnetic radiation, and concluded that the dynamic behaviors of the neural network could be adjusted by changing the number of neurons in the neural network that affected by electromagnetic radiation. The theoretical analyses were verified by circuit experiments, which gave a new understanding to the pathogenesis of neurological diseases ${ }^{[29,30]}$. Moreover, recently, Allehiany et al. ${ }^{[31]}$ used the adaptive sliding mode control technique to investigate a fractional order neural network under electromagnetic radiation, and Yu et al. ${ }^{[32]}$ designed a pseudo-random number generator based on a $\mathrm{HNN}$ under electromagnetic radiation. However, all these works about electromagnetic radiation set the bias current of the neurons to zero or the bias current of only one neuron to not zero. In fact, the bias current can improve the flexibility and fitness of the neural network $^{[33,34]}$. Therefore, it is necessary to study a HNN with multiple or dual bias currents that affected by electromagnetic radiation.

Motivated by the above mentioned considerations, in this paper, we focus on a HNN composed of three neurons, in which the external electromagnetic radiation is imposed on one of the three neurons and the remaining two neurons are added with bias currents. What is more, we use the fluctuation of magnetic flux to emulate the influence of electromagnetic radiation on the electrical activities of the neuron, and utilize a flux-controlled memristor to describe the feedback of magnetic flux on membrane voltage. In order to explore the dynamical behaviors of the HNN, we conduct Matlab theoretical analyses, and perform Multisim simulations and hardware experiments for circuit-level verification. As a consequence, the results show that in a neural network with electromagnetic radiation and dual bias currents, the neural network will exhibit complex and fantastic dynamic behaviors, such as coexisting periodic attractor, quasi-periodic attractor, chaotic attractor and transient chaotic attractor. Moreover, a series of transient chaotic attractors with different chaotic times are also captured. These coexisting attractors show that this kind of neural network has a rich multistability. Meanwhile, we find parallel bifurcation behaviors, which have hardly been reported in the articles about electromagnetic radiation. To a certain extent, this work may shed light 
on some cerebral pathologies and is worthy of further detailed investigation.

The remaining sections of this paper are organized as follows. In Sec. 2, the neural network model with dual bias currents affected by electromagnetic radiation is established, and the basic properties including boundary, equilibrium point and symmetry are discussed carefully. In Sec. 3, the dynamics of the neural network are studied in detail with the help of Matlab numerical analyses. And an appropriate electronic circuit is proposed in Sec. 4 by Multisim simulation and hardware experiment. Finally, the conclusions are summarized in Sec. 5 .

\section{Description of the proposed Hopfield neural network}

\subsection{The mathematical model}

A generic Hopfield neural network containing n neurons can be expressed by a set of circuit equations ${ }^{[14]}$, for the $i$-th neuron is expressed as

$$
C_{i} \frac{d x_{i}}{d t}=-\frac{x_{i}}{R_{i}}+\sum_{j=1}^{n} w_{i j} \tanh \left(x_{j}\right)+I_{i}
$$

$x_{i}$ is the membrane voltage of the $i$-th neuron, $R_{i}$ and $C_{i}$ are the membrane resistance and capacitance between the inside and outside of the neuron, $\tanh \left(x_{j}\right)$ is the activation function representing the voltage input from the $j$-th neuron, $w_{i j}$ is the synapse weight illustrating the strength of the connection between the $i$-th neuron and the $j$-th neuron, and $I_{i}$ is the input bias current.

In this paper, we consider a three-neuron-based HNN, what's more, the second neuron is exposed to electromagnetic radiation, and the input dual bias currents are set as

$$
I_{i}=\left[\begin{array}{lll}
I_{1} & I_{2} & I_{3}
\end{array}\right]^{T}=\left[\begin{array}{lll}
I & 0 & I
\end{array}\right]^{T}
$$

After a lot of experiments, the connection topology of the neural network in this paper is selected as

$$
w=\left[\begin{array}{lll}
w_{11} & w_{21} & w_{31} \\
w_{12} & w_{22} & w_{32} \\
w_{13} & w_{23} & w_{33}
\end{array}\right]=\left[\begin{array}{ccc}
1.5 & 2 & 0.9 \\
-1.5 & 1.5 & 0 \\
3 & -2 & 0.8
\end{array}\right]
$$

Different from the connection weight in other Hopfield neural networks, the neural network studied in this paper is a new neural network.

For a neuron, when it is exposed to electromagnetic radiation, its membrane magnetic flux will change. Therefore, we can add the magnetic flux as a new variable to describe the effect of electromagnetic 
radiation on the neuron, and employ a flux-controlled memristor to describe the feedback of magnetic flux on membrane voltage. The topology of the HNN is shown in Fig. 1. Consequently, when $C_{1}=C_{2}=C_{3}=1$ and $R_{1}=R_{2}=R_{3}=1$, the HNN with dual bias currents affected by electromagnetic radiation can be described as follows

$$
\left\{\begin{array}{l}
\frac{d x_{1}}{d t}=-x_{1}+1.5 \tanh \left(x_{1}\right)+2 \tanh \left(x_{2}\right)+0.9 \tanh \left(x_{3}\right)+I \\
\frac{d x_{2}}{d t}=-x_{2}-1.5 \tanh \left(x_{1}\right)+1.5 \tanh \left(x_{2}\right)+k_{1}\left(a+3 b \varphi^{2}\right) x_{2} \\
\frac{d x_{3}}{d t}=-x_{3}+3 \tanh \left(x_{1}\right)-2 \tanh \left(x_{2}\right)+0.8 \tanh \left(x_{3}\right)+I \\
\frac{d \varphi}{d t}=k_{2} x_{2}
\end{array}\right.
$$

Where $\varphi$ is the magnetic flux of the second neuron cell membrane, $a+3 b \varphi^{2}$ denotes the memconductance of the flux-controlled memristor, $a$ and $b$ are the electromagnetic radiation parameters, $k_{1}$ represents the strength of external electromagnetic radiation, and $k_{2}$ indicates the influence of the second membrane potential on magnetic flux.

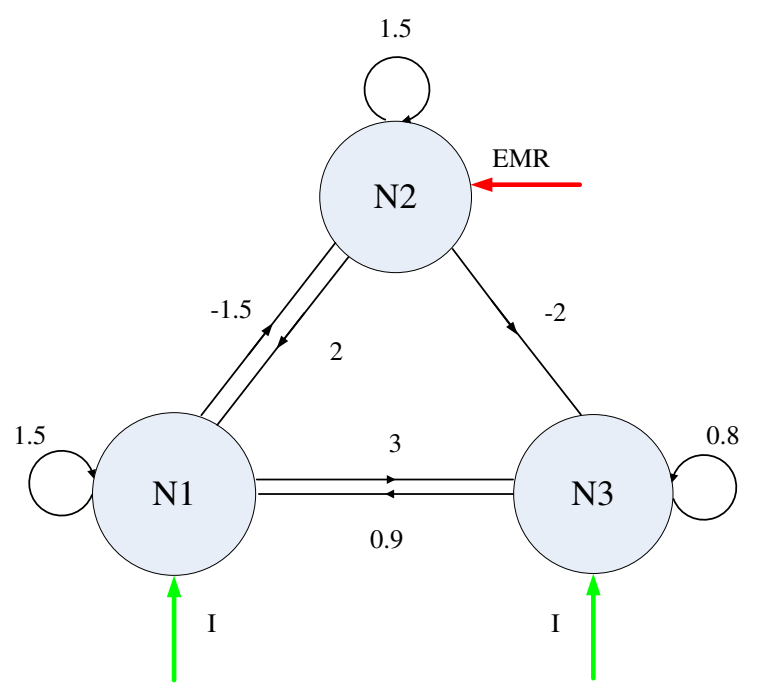

Fig. 1 The topological connection of the proposed HNN under electromagnetic radiation and dual bias currents.

\subsection{Proof of boundary}

It can be proved that the orbits of the Eq. (4) are not infinitely divergent, but always confined to a boundary area. Referring the approach of Ref. [35, 36], a Lyapunov function is proposed as follows

$$
V\left(x_{1}, x_{2}, x_{3}, x_{4}\right)=\frac{1}{2}\left(x_{1}^{2}+x_{2}^{2}+x_{3}^{2}+\varphi^{2}\right)
$$

Differentiating Eq. (5) in the time domain yields 


$$
\begin{aligned}
\dot{V}\left(x_{1}, x_{2}, x_{3}, \varphi\right)= & x_{1} \dot{x}_{1}+x_{2} \dot{x}_{2}+x_{3} \dot{x}_{3}+\varphi \dot{\varphi} \\
= & -x_{1}^{2}+1.5 x_{1} \tanh \left(x_{1}\right)+2 x_{1} \tanh \left(x_{2}\right)+0.9 x_{1} \tanh \left(x_{3}\right)+I x_{1} \\
& -x_{2}^{2}-1.5 x_{2} \tanh \left(x_{1}\right)+1.5 x_{2} \tanh \left(x_{2}\right)+k_{1}\left(a+3 b \varphi^{2}\right) x_{2}^{2} \\
& -x_{3}^{2}+3 x_{3} \tanh \left(x_{1}\right)-2 x_{3} \tanh \left(x_{2}\right)+0.8 x_{3} \tanh \left(x_{3}\right)+I x_{3} \\
& +k_{2} x_{2} \varphi
\end{aligned}
$$

In order to facilitate calculations, defining

$$
\begin{aligned}
v\left(x_{1}, x_{2}, x_{3}\right)= & \left(1.5 x_{1}-1.5 x_{2}+3 x_{3}\right) \tanh \left(x_{1}\right) \\
& +\left(2 x_{1}+1.5 x_{2}-2 x_{3}\right) \tanh \left(x_{2}\right) \\
& +\left(0.9 x_{1}+0.8 x_{3}\right) \tanh \left(x_{3}\right)
\end{aligned}
$$

For all $x \in R$, it is well known that $-1<\tanh (x)<1$. Therefore, Eq. (7) can be simplified as

$$
\begin{aligned}
v\left(x_{1}, x_{2}, x_{3}\right) \leq & \left|\left(1.5 x_{1}-1.5 x_{2}+3 x_{3}\right) \tanh \left(x_{1}\right)\right| \\
& +\left|\left(2 x_{1}+1.5 x_{2}-2 x_{3}\right) \tanh \left(x_{2}\right)\right| \\
& +\left|\left(0.9 x_{1}+0.8 x_{3}\right) \tanh \left(x_{3}\right)\right| \\
< & \left|1.5 x_{1}-1.5 x_{2}+3 x_{3}\right|+\left|2 x_{1}+1.5 x_{2}-2 x_{3}\right|+\left|0.9 x_{1}+0.8 x_{3}\right| \\
\leq & 4.4\left|x_{1}\right|+3\left|x_{2}\right|+5.8\left|x_{2}\right|
\end{aligned}
$$

Making $D_{0}>0$ to the sufficiently large area, for all $\left(x_{1}, x_{2}, x_{3}, \varphi\right)$ that satisfying $V\left(x_{1}, x_{2}, x_{3}, \varphi\right)=D$ with $D>D_{0}$, it can be concluded that

$$
v\left(x_{1}, x_{2}, x_{3}\right)<4.4\left|x_{1}\right|+3\left|x_{2}\right|+5.8\left|x_{3}\right|<x_{1}^{2}+x_{2}^{2}+x_{3}^{2}
$$

Then Eq. (6) can be simplified as

$$
\begin{aligned}
\dot{V}\left(x_{1}, x_{2}, x_{3}, \varphi\right)= & -x_{1}^{2}-x_{2}^{2}-x_{3}^{2} \\
& +I x_{1}+I x_{3}+k_{1}\left(a+3 b \varphi^{2}\right) x_{2}^{2}+k_{2} \varphi x_{2} \\
& +v\left(x_{1}, x_{2}, x_{3}\right) \\
< & I x_{1}+I x_{3}+k_{1}\left(a+3 b \varphi^{2}\right) x_{2}{ }^{2}+k_{2} \varphi x_{2}
\end{aligned}
$$

According to Ref. [37], in the situation all $\left(x_{1}, x_{2}, x_{3}, \varphi\right)$ that contenting $V\left(x_{1}, x_{2}, x_{3}, \varphi\right)=D$ with $D>D_{0}$, the below requirement exists

$$
k_{1}\left(a+3 b \varphi^{2}\right) x_{2}^{2}+k_{2} \varphi x_{2}<-\left(I x_{1}+I x_{3}\right)
$$

Putting Eq. (11) into Eq. (10), there is

$$
\dot{V}\left(x_{1}, x_{2}, x_{3}, \varphi\right)<0
$$

As a Consequence, it implies that on the surface

$$
\left\{\left(x_{1}, x_{2}, x_{3}, \varphi\right) \mid V\left(x_{1}, x_{2}, x_{3}, \varphi\right)=D\right\}
$$

Then set 


$$
\left\{\left(x_{1}, x_{2}, x_{3}, \varphi\right) \mid V\left(x_{1}, x_{2}, x_{3}, \varphi\right) \leq D\right\}
$$

It means that the trajectories of the system are limited to a bounded area.

\subsection{Equilibrium point and symmetry}

System attractors are divided into self-excited attractors and hidden attractors. From the perspective of attraction basin and equilibrium points, the attraction basin of the self-excited attractors contain the unstable equilibrium points, but the attraction basin of the hidden attractors does not intersect with any unstable equilibrium points. According to Ref. [38], four types of systems with hidden attractors are classified as: (1) the system does not have any equilibrium points, (2) the system has only stable equilibrium points, (3) the equilibrium points of the system are curves, and (4) the equilibrium points of the system are surfaces.

Letting the left side of Eq. (4) equals to zero, the equilibrium points of the proposed HNN can be solved by

$$
\left\{\begin{array}{c}
-x_{1}+1.5 \tanh \left(x_{1}\right)+2 \tanh \left(x_{2}\right)+0.9 \tanh \left(x_{3}\right)+I=0 \\
-x_{2}-1.5 \tanh \left(x_{1}\right)+1.5 \tanh \left(x_{2}\right)+k_{1}\left(a+3 b \varphi^{2}\right) x_{2}=0 \\
-x_{3}+3 \tanh \left(x_{1}\right)-2 \tanh \left(x_{2}\right)+0.8 \tanh \left(x_{3}\right)+I=0 \\
k_{2} x_{2}=0
\end{array}\right.
$$

It is obviously that $x_{2}$ is equal to zero, so in the feedback item of external electromagnetic radiation $k_{1}\left(a+3 b \varphi^{2}\right) x_{2}, \varphi$ can be setted to any value. Putting $x_{2}=0$ into Eq. (15), then Eq. (15) can be simplified to

$$
\left\{\begin{array}{c}
-x_{1}+1.5 \tanh \left(x_{1}\right)+0.9 \tanh \left(x_{3}\right)+I=0 \\
-1.5 \tanh \left(x_{1}\right)=0 \\
-x_{3}+3 \tanh \left(x_{1}\right)+0.8 \tanh \left(x_{3}\right)+I=0
\end{array}\right.
$$

From Eq. (16), $x_{1}=0$ can be deduced, then Eq. (16) can be simplified to

$$
\left\{\begin{array}{c}
0.9 \tanh \left(x_{3}\right)+I=0 \\
-x_{3}+0.8 \tanh \left(x_{3}\right)+I=0
\end{array}\right.
$$

It can be easily concluded that there is only one solution in Eq. (17), that is

$$
\left\{\begin{array}{l}
x_{3}=0 \\
I=0
\end{array}\right.
$$

But in this paper, there are dual bias currents in the first neuron and third neuron of the HNN, that is to say, $I \neq 0$. So the neural network (4) has no equilibrium points, and all attractors in the HNN are hidden attractors. 
To judge whether a system has symmetry, it depends on whether the solutions of the system remain unchanged under the symmetric transformation. Similarly, due to the presence of the dual bias current $I$, the proposed HNN is not symmetrical about any coordinate axes, because the HNN does not remain invariant under the any transformation. Thus, it can be seen that the attractors of the proposed HNN are more intriguing and singular.

\section{Complex dynamic analyses of the proposed Hopfield neural network}

This section mainly discusses the dynamic behaviors of the proposed HNN. The research methods include phase portrait, time domain waveform, bifurcation diagram and Lyapunov exponent, etc. The phase portrait is the most intuitive method of observing the dynamic behaviors, which is the record of the trajectory of the system. The time domain waveform describes the change of the signal over the time, which can be a good verification of the phase portrait. The bifurcation diagram can clearly describe the characteristics of the system performance changing with the system parameters. In this article, taking the local maxima of the variable is used to obtain the bifurcation diagram. The Lyapunov exponent is also an indispensable research method. Its positive or negative value along a certain direction can represent the average divergence or convergence of the adjacent orbits in the attractor along that direction. Remark that the below numerical simulations are all use fourth order Runge-Kutta algorithm.

\subsection{Transient chaos}
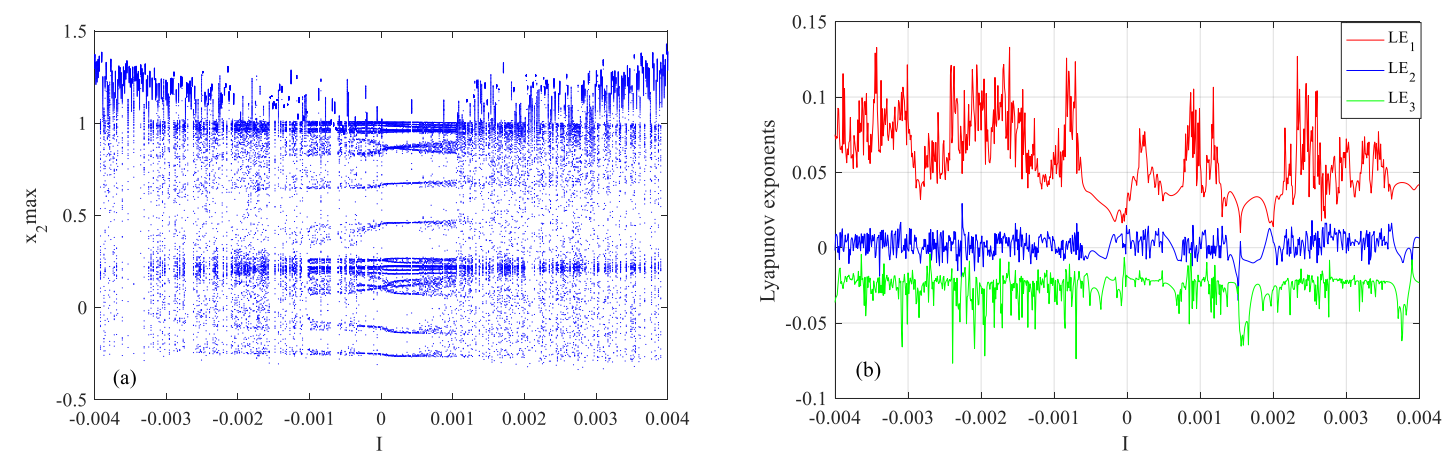

Fig. 2 Dynamics for $\mathrm{I} \in[-0.004,0.004]$, (a) is the bifurcation diagram; (b) is the corresponding first three Lyapunov exponents.

Transient chaos is a special phenomenon in chaotic dynamics. Transient chaos refers to the chaotic behaviors of the system on a finite time scale, which ultimately evolves into regular behaviors such as fixed point or periodic orbit ${ }^{[39,40]}$. From the topological point of view, transient chaos is caused by chaotic saddle point in the phase space. When the attractor intersects with the boundary of the attraction basin, the chaotic saddle point appears, and then the chaotic attractor encounters a chaotic crisis. The chaotic attractor 
disappears and the system trajectories escape to the external attractor, then the chaotic attractor is replaced by the periodic attractor. The duration of chaotic behaviors strongly depend on the system parameters and initial conditions, and a small difference will cause different chaotic times. Next, transient chaotic behaviors in this HNN will be explored.
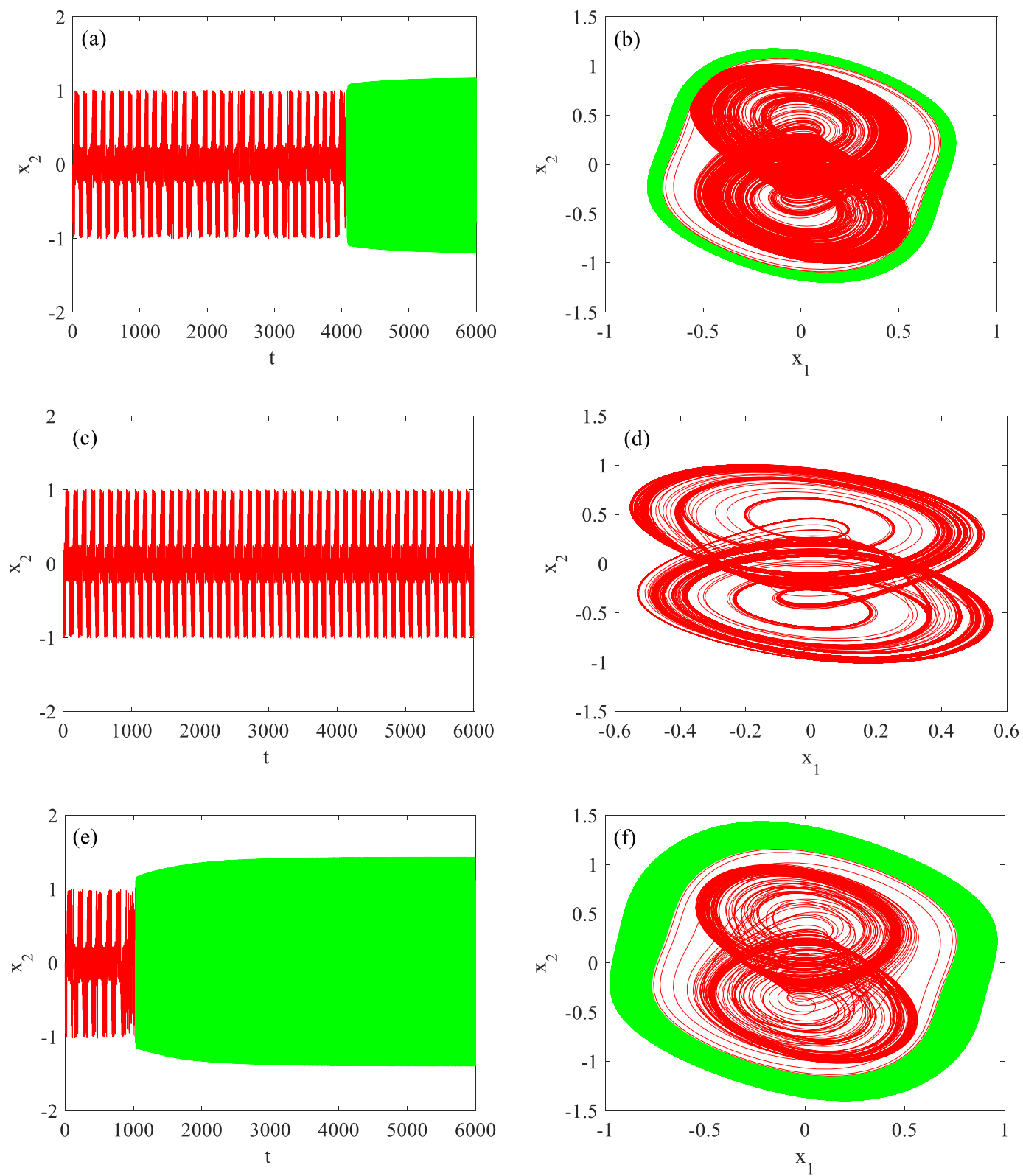

Fig. 3 Transient chaotic attractors and chaotic attractor with different $I$. (a) and (b), $I=-0.0018$; (c) and (d), $I=0.0003$; (e) and (f), $I=0.0035$.

Firstly, setting the bias current $I$ as the dynamical parameter, and the range of change is $-0.004 \leq I \leq 0.004$. The other parameters are fixed as $a=1.5, b=-0.05, k_{1}=-0.3$ and $k_{2}=-0.1$. As for system initial conditions, they are $\left(x_{10}, x_{20}, x_{30}, \varphi_{0}\right)=(0.1,0,0.1,0.1)$. The bifurcation diagram of $x_{2}$ varing with $I$ is shown in Fig. 2(a), and the corresponding first three Lyapunov exponents are displayed in 
Fig. 2(b). From Fig. 2(a), we can see that in the region $-0.004 \leq I \leq 0.004$, most of the bifurcation behaviors of the HNN are dense and irregular points, which indicates that the system state is always in chaos. But it is worth noting that in the range of $-0.0009 \leq I \leq 0.0009$, the bifurcation behaviors are different, that is to say, the system state occurs transformation. After a large number of time domain waveform and phase portrait experiments, we find that as the bias current $I$ slowly increase, the system evolves from transient chaotic behaviors to chaotic behaviors and finally back to transient chaotic behaviors. These complex dynamic behaviors are caused by boundary crisis. Combined with the Lyapunov exponents in Fig. 2(b), it can be seen that the maximum Lyapunov exponent is always greater than zero under this condition, which is different from that the maximum Lyapunov exponent equals to zero in quasi-periodic behaviors.

In order to get insight into these dynamic behaviors more directly, the phase portraits and corresponding time domain waveforms are given in Fig. 3 when $t \in(0,6000)$. For representative, $I=-0.0018$ in the region $-0.004 \leq I \leq-0.0009, \quad I=0.0003$ in the region $-0.0009 \leq I \leq 0.0009$ and $I=0.0035$ in the region $0.0009 \leq I \leq 0.004$ are selected. Obviously, Fig. 3(a) represents chaotic behaviors in the time region $t \in(0,4083)$, and then turns into periodic behaviors. Fig. 3(c) always maintains at chaotic behaviors. As for Fig. 3(e), it holds chaotic behaviors in the time region $t \in(0,1038)$, and then settles down on periodic behaviors. What's more, Fig. 3(b), Fig. 3(d) and Fig. 3(f) are the phase portraits of Fig. 3(a), Fig. 3(c) and Fig. 3(e) respectively. In Fig. 3, the red curves represent chaotic behaviors and the green curves represent periodic behaviors.

Due to that the transient chaos is a kind of unstable behavior during the transformation process from period to chaos with random changes in a short or long time. After further research, it is found that the transient chaotic behaviors are not only related to the value of the bias current $I$, but also associated with the system initial value $\varphi_{0}$. That is to say, for different $\varphi_{0}$, the HNN can generate transient chaotic behaviors with different chaotic times. When $a=1.5, b=-0.05, k_{1}=-0.3, k_{2}=-0.1, I=-0.001$, system initial conditions $\left(x_{10}, x_{20}, x_{30}\right)=(0.1,0,0.1)$ and $t \in(0,6000)$, Fig. 4 exhibits transient chaotic behaviors with different chaotic times under different values of $\varphi_{0}: \varphi_{0}=-0.03, \varphi_{0}=0.002$ and $\varphi_{0}=-0.1$. Remark that the neural network in this paper can display multiple transient chaotic attractors with different chaotic times at different initial values of $\varphi_{0}$, and only a few typical examples are represented for demonstration in Fig 4. Similarly, Fig. 4(a), Fig. 4(c) and Fig. 4(e) are the time domain waveforms, and Fig. 4(b), Fig. 4(d) and Fig. 4(f) are the corresponding phase portraits. The chaotic behaviors are colored in red 
and the periodic behaviors are colored in green.
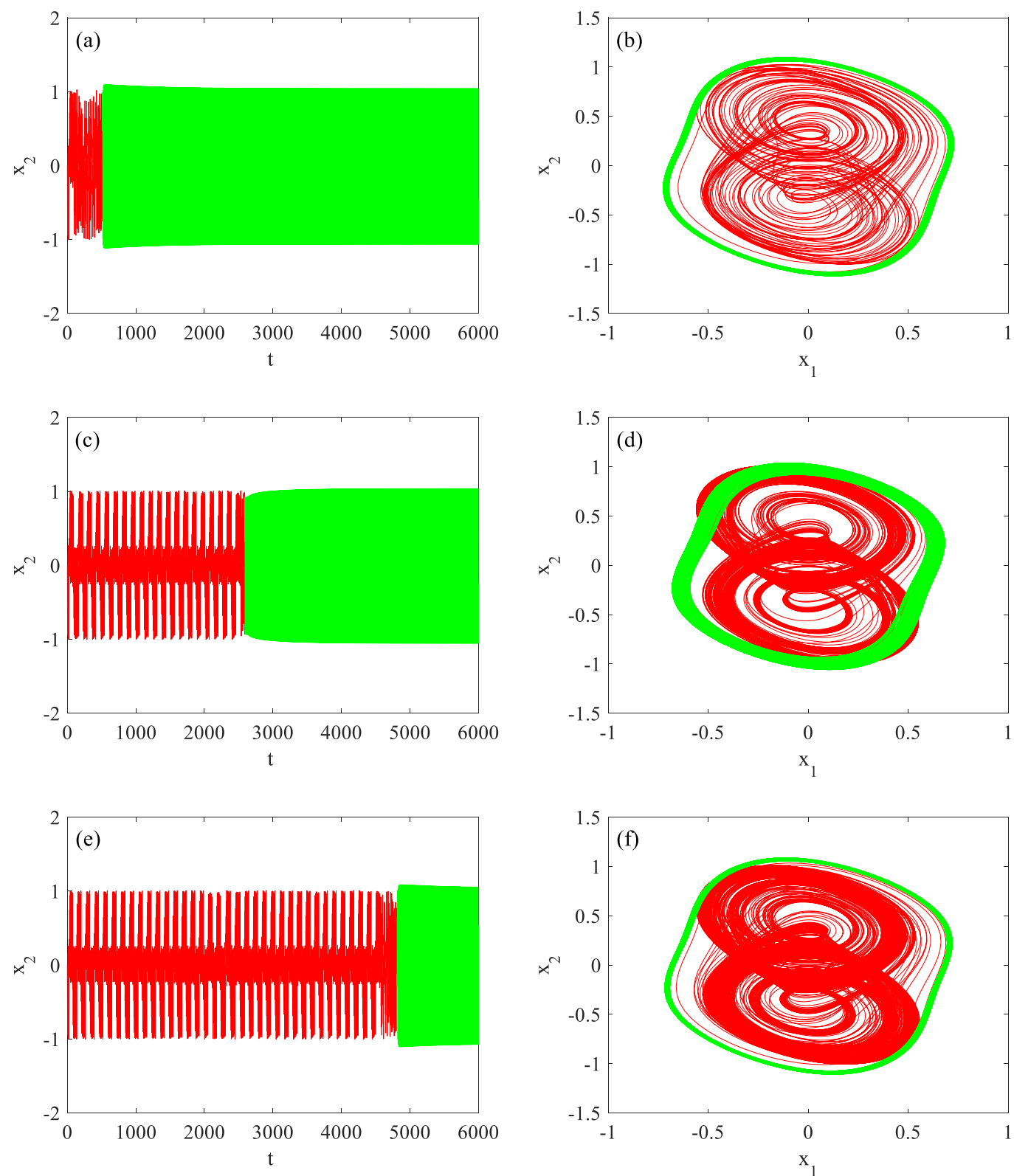

Fig. 4 Coexisting transient chaotic attractors with different chaotic times, (a) and (b), $\varphi_{0}=-0.03$; (c) and (d), $\varphi_{0}=0.002$; (e) and (f), $\varphi=-0.1$.

\subsection{Parallel bifurcation}

In recent years, a new phenomenon has been discovered, named parallel bifurcation ${ }^{[41-43]}$. This phenomenon means that the system has multiple bifurcation behaviors at the same time under the same parameters and initial conditions. To observe the influence of the second neuron membrane potential on magnetic flux, let $k_{2}$ increase monotonously from -0.8 to 0.8 and other parameters are kept as $a=1.5, b=-0.05, \quad k_{1}=-0.3$ and $I=-0.001$, Fig. 5 reveals its abundant dynamic behaviors under initial conditions $\left(x_{10}, x_{20}, x_{30}, \varphi_{0}\right)=(0.1,0,0.1,0.1)$. In Fig. $5(\mathrm{a})$, it can be seen that there are periodic doubling 
bifurcation and reverse periodic doubling bifurcation behaviors in the region $-0.5<k_{2}<-0.25$ and $0.25<k_{2}<0.5$. For details, Fig. 6 is the enlargement of the bifurcation diagram of Fig. 5(a).
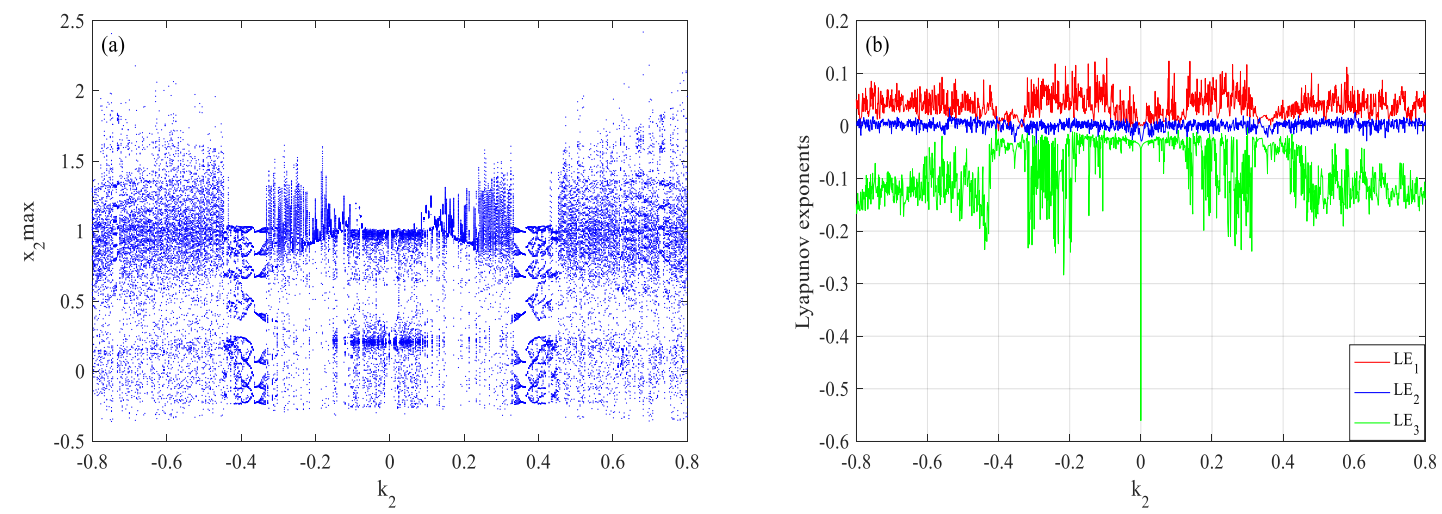

Fig. 5 Dynamics for $\mathrm{k}_{2} \in[-0.8,0.8]$, (a) is the bifurcation diagram; (b) is the corresponding first three Lyapunov exponents.
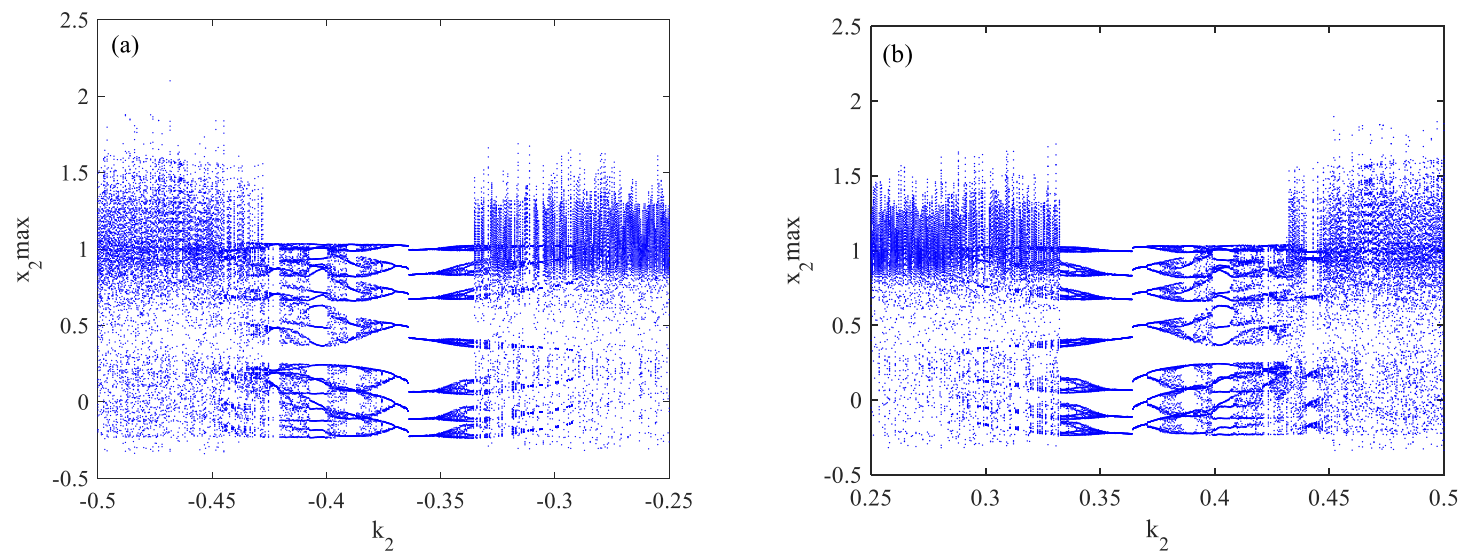

Fig. 6 Extended bifurcation diagrams of Fig. 5(a), (a) $\mathrm{k}_{2} \in[-0.5,-0.25]$; (b) $\mathrm{k}_{2} \in[0.25,0.5]$.

In Fig. 6(a), when $k_{2}=-0.3627$, the system attractor is quasi-periodic attractor, and then each of its orbits occurs periodic doubling bifurcation and reverse periodic doubling bifurcation behaviors. In the chaotic region $-0.5<k_{2}<-0.3763$, some quasi-periodic windows are existed, for example $-0.4248<k_{2}<-0.4188,-0.4068<k_{2}<-0.3993$ and $-0.3908<k_{2}<-0.3858$. In these regions, the bifurcations are complicated but regular. Meanwhile, in Fig. 6(b), $k=0.3652$ is the critical value. At this value, each of the orbits of the quasi-periodic attractor of the system produces periodic doubling bifurcation and reverse periodic doubling bifurcation behaviors as well. Likewise, in the chaotic domains $0.3783<k_{2}<0.5$, some quasi-periodic windows are sandwiched, like $0.3863<k_{2}<0.3918$, $0.3988<k_{2}<0.4093$ and $0.4218<k_{2}<0.4243$. In order to better observe such complex dynamics, the numerical results of the first three Lyapunov exponents in Fig. 5(b) verifies these rich dynamic behaviors. That is to say, in most of the region $-0.8<k_{1}<0.8$, the first three Lyapunov exponents are a positive value, a null value and a negative value, which indicating that the system attractor is chaotic attractor. However, in 
the quasi-periodic regions, such as $-0.3752<k_{2}<-0.3432$ and $0.3532<k_{2}<0.3768$, the first three Lyapunov exponents are two null values and a negative value. Undoubtedly, through changing $k_{2}$ in small steps, the HNN can appear unusual irregular behaviors and regular behaviors, which may be of great significance to the treatment of neurological diseases such as Alzheimer and Psychosis.

Coincidentally, similar parallel bifurcation behaviors also occur if the parameter $b$ changes. When $a=1.5, k_{1}=-0.3, k_{2}=-0.1, I=-0.001$ and $\left(x_{10}, x_{20}, x_{30}, \varphi_{0}\right)=(0.1,0,0.1,0.1)$ are chosen, the parameter $b$ bifurcation diagram is given in Fig. 7. In sight of Fig. 7, compared with Fig. 5(a), when $b=-0.6333$, the system is also a quasi-periodic attractor, but it just undergoes a series of reverse periodic doubling scenario to chaos and no forward periodic doubling scenario. Instead, as parameter $b$ increases, when it reaches -0.5812 , every orbits of the quasi-attractor breaks into chaotic state through tangent bifurcation, and finally keep in chaotic state except some quasi-periodic mini-zones at $b=-0.517, b=-0.485, b=-0.4649$ and $b=-0.4188$. It is stressed that in the dynamic behaviors mentioned above, there are some noises, which leads to some irregular points in addition to the normal reverse period doubling bifurcation and tangent bifurcation processes. In fact, this phenomenon of noises may be better consistent with the firing activities in the real human brain dynamics. Because from the physical point of view, according to Ref. [44], even if the energy cannot be completely absorbed, the participation of noises can also input energy for the system.

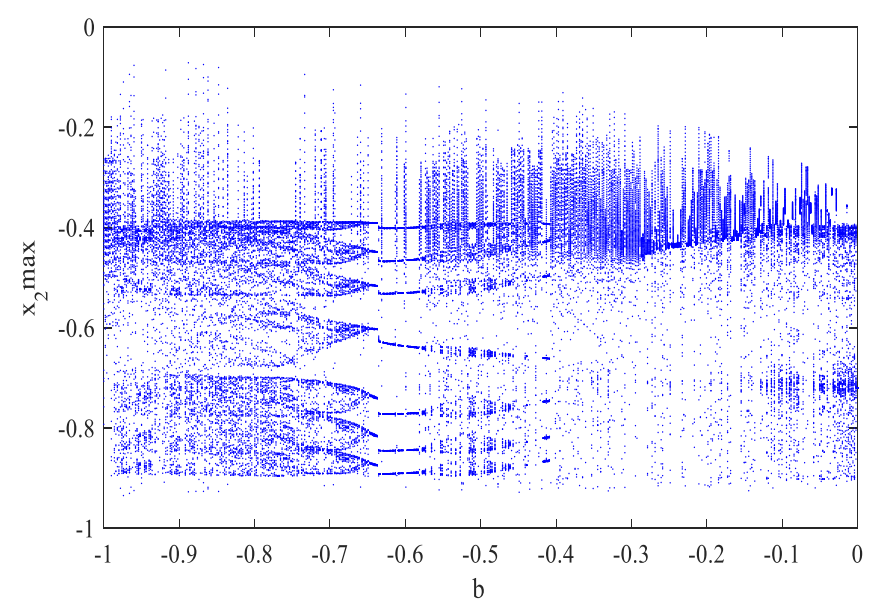

Fig. 7 Bifurcation diagram for $\mathrm{b} \in[-1,0]$.

According to the above discussion, to the best of the author's knowledge, the parallel bifurcation is rare in other systems, especially in the Hopfield neural network. Hence, this kind of dynamic phenomenon has certain research significance and is worth exploring.

\subsection{Coexisting attractors}


Coexistence of attractors is a kind of special and interesting characteristic in nonlinear dynamics, which means that the system has different dynamic phenomenon under the same set of parameters but different initial conditions. This feature provides great flexibility for engineering applications and is a research hotspot for scholars. As a nonlinear system, the coexisting attractors of neural network are also the focus of research. The next part reveals the pattern of the coexisting attractors in this neural network.
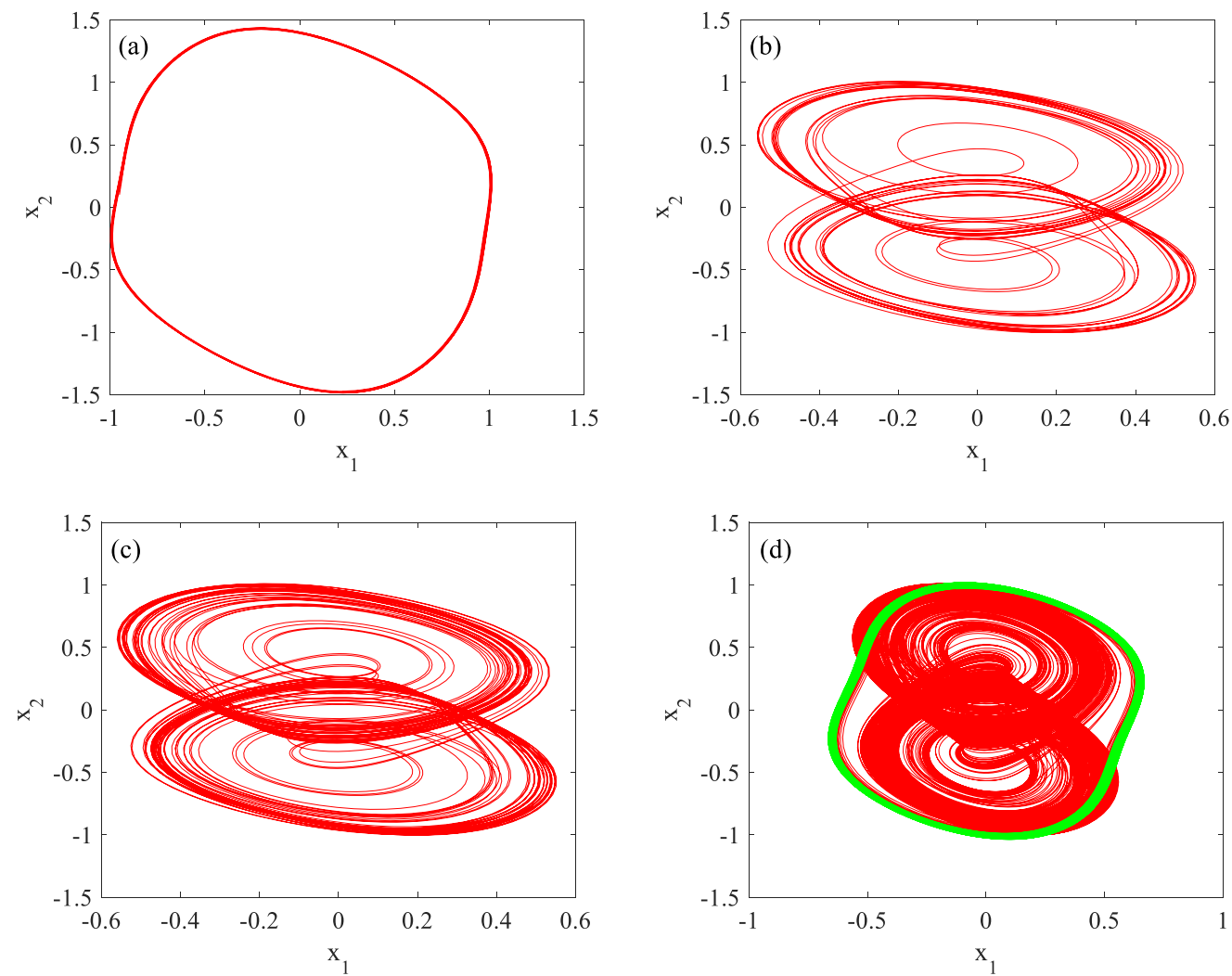

Fig. 8 Coexisting attractors under different initial conditions, (a) periodic attractor for $(-0.95,0.1,0.09,-2.45)$; (b) quasi-periodic attractor for $(-0.1,-0.1,0,0.1) ;$ (c) chaotic attractor for $(-0.1,-0.1,-0.1,-0.1) ;$ (d) transient chaotic attractor for $(0.1,0.1,0,0.1)$.

When the parameters are assigned with appropriate values: $a=1.5, b=-0.05, k_{1}=-0.3, k_{2}=-0.1$ and $I=-0.001$, for different sets of initial conditions, four kinds of attractors can be triggered in this neural network as shown in Fig. 8, which depicts the phase portraits of the attractors on the $\mathrm{x}_{1}-\mathrm{x}_{2}$ plane. In Fig. 8(a), the neural network starts from the initial conditions $\left(x_{10}, x_{20}, x_{30}, \varphi_{0}\right)=(-0.95,0.1,0.09,-2.45)$. Obviously, it is a periodic attractor which means the dynamics are steady. In order to comprehensively analyze this behavior, its Lyapunov exponents are computed as $L E_{1}=0.00191, L E_{2}=-0.02914$, $L E_{3}=-0.43091$ and $L E_{4}=-0.76618$, which the maximum Lyapunov exponent is about to zero. And undoubtedly, this regular behavior has large impact on the cure for neurological diseases. In Fig. 8(b), it can be obtained that the attractor is in quasi-periodic state when the initial conditions are chosen as $\left(x_{10}, x_{20}, x_{30}, \varphi_{0}\right)=(-0.1,-0.1,0,0.1)$. As for its Lyapunov exponents, they are $L E_{1}=0.00182$, 
$L E_{2}=0.00123, L E_{3}=-0.03988$ and $L E_{4}=-0.49332$. In this quasi-periodic case, the system is in a transition state from period to chaos. And then, under the initial conditions $\left(x_{10}, x_{20}, x_{30}, \varphi_{0}\right)=(-0.1,-0.1,-0.1,-0.1)$, a chaotic attractor can be produced as shown in Fig. 8(c), which the Lyapunov exponents are $L E_{1}=0.02739, L E_{2}=0.00726, L E_{3}=-0.04391$ and $L E_{4}=-0.50371$. Moreover, transient chaotic attractor can be also captured as drawn in Fig. 8(d) with the initial conditions $\left(x_{10}, x_{20}, x_{30}, \varphi\right)=(0.1,0.1,0,0.1)$. In Fig. 8(d), chaotic orbits are shown in red and periodic orbits are shown in green. The orbits of the transient chaotic attractor exhibits chaotic motion in the time interval $t \in(0,2600)$, and subsequently degrades into periodic oscillations. As for its Lyapunov exponents, they are $L E_{1}=0.05024, L E_{2}=-0.00159, L E_{3}=-0.41329$ and $L E_{4}=-0.52077$.

Based on the above discussions, we know that there are a variety of coexisting hidden attractors in the neural network, including periodic and chaotic attractors in the steady state, and quasi-periodic and transient chaotic attractors in the switching state. This phenomenon shows that the system is very sensitive to the initial values, that is, the initial values have great influence on the dynamic behaviors of the neural network. From the perspective of practical applications, it provides new ideas for solving potential and unpredictable events.

\section{Circuit realization}

Realizing the circuit of the mathematical model through commercially available discrete electrical components is a very necessary step in the study of the dynamics of neural network. Moreover, the circuit realization based on the mathematical model is essential for practical engineering applications, which can reflect the feasibility of the theoretical model. In this section, a circuit is designed by the Multisim and breadboard to support the previous theoretical calculations.

Before constructing the neural network circuit, we first introduce the nonlinear activation function $-\tanh (\cdot)$, which is a hyperbolic tangent function, is shown in Fig. 9. In Ref. [45, 46], a pair of bipolar NPN transistors, two operational amplifiers, eight resistors, a constant current source $I_{0}$ and a supply voltage $V_{C C}$ are utilized to implement the hyperbolic tangent function module. When the component parameters are selected as $R=10 \mathrm{k} \Omega, R_{F}=520 \Omega, R_{C}=1 \mathrm{k} \Omega, V_{C C}=15 \mathrm{~V}$ and $I_{0}=1.1 \mathrm{~mA}$, the input-output equation can be written as $v_{0}=-\tanh \left(v_{i}\right)$. In addition, according to Ref. $[47,48]$, the constant current source $I_{0}$ can be replaced by the circuit with a pair of bipolar NPN transistors, three resistors and a supply voltage $V_{E E}$ in the solid line box of Fig. 9 , where the corresponding parameters are set as $R_{w}=9.8 k \Omega, R_{T}=2 k \Omega$ and 
$V_{E E}=-15 \mathrm{~V}$

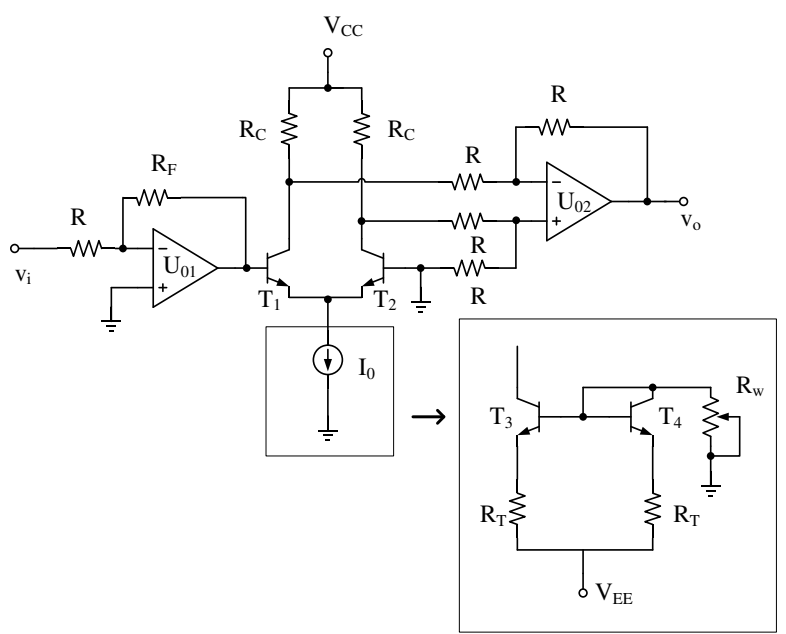

Fig. 9 Circuit realization of hyperbolic tangent function.

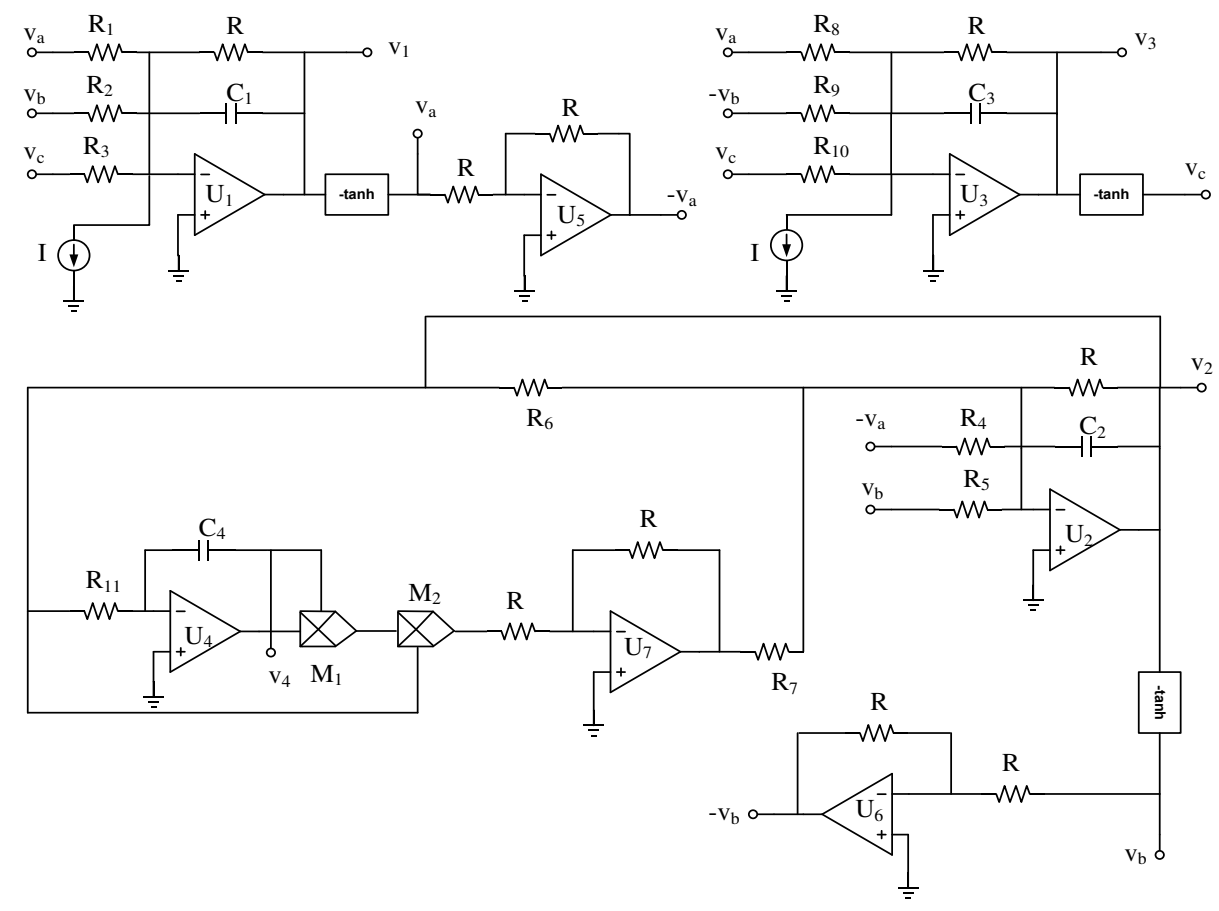

Fig. 10 Circuit scheme of the proposed neural network.

With the proposed model of Eq. (4), the three-neuron-based neural network under electromagnetic radiation and dual bias currents can be physically synthesized as shown in Fig. 10, where the circuit modules inside the solid line boxes marked by - tanh are the inverting hyperbolic tangent function circuit units plotted in Fig. 9. There are four dynamical capacitors in the required circuit of Fig. 10, where the capacitor voltages $v_{1}, v_{2}, v_{3}$ and $v_{4}$ denote the state variables $x_{1}, x_{2}, x_{3}$ and $\varphi$ respectively. Hence, by employing Kirchhoff's circuit laws in Fig. 10, the circuit equations are formulated as 


$$
\left\{\begin{array}{l}
R C \frac{d v_{1}}{d t}=-v_{1}+\frac{R}{R_{1}} \tanh \left(v_{1}\right)+\frac{R}{R_{2}} \tanh \left(v_{2}\right)+\frac{R}{R_{3}} \tanh \left(v_{3}\right)+I \\
R C \frac{d v_{2}}{d t}=-v_{2}-\frac{R}{R_{4}} \tanh \left(v_{1}\right)+\frac{R}{R_{5}} \tanh \left(v_{2}\right)+R\left(-\frac{1}{R_{6}}+\frac{v_{4}^{2}}{R_{7}}\right) v_{2} \\
R C \frac{d v_{3}}{d t}=-v_{3}+\frac{R}{R_{8}} \tanh \left(v_{1}\right)-\frac{R}{R_{9}} \tanh \left(v_{2}\right)+\frac{R}{R_{10}} \tanh \left(v_{3}\right)+I \\
R C \frac{d v_{4}}{d t}=-\frac{R}{R_{11}} v_{2}
\end{array}\right.
$$

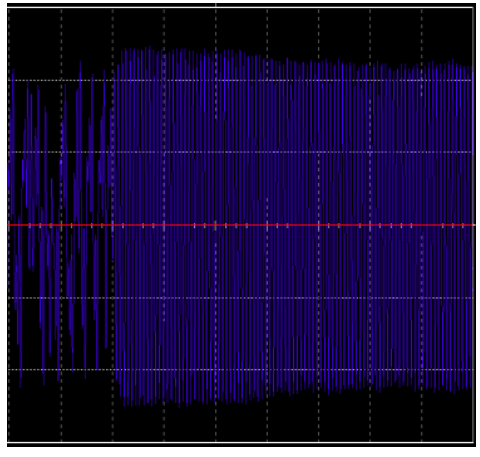

(a)

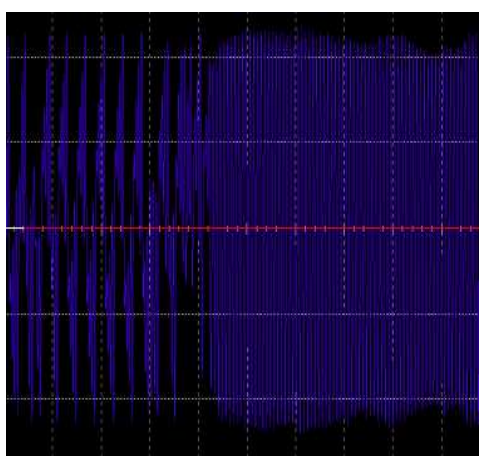

(c)

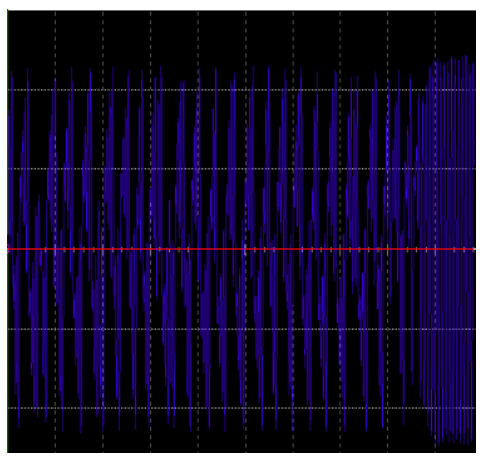

(e)

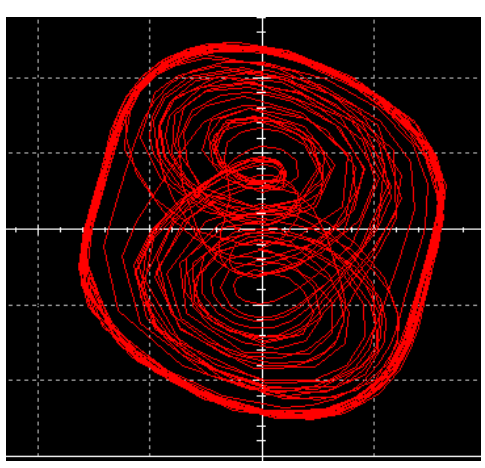

(b)

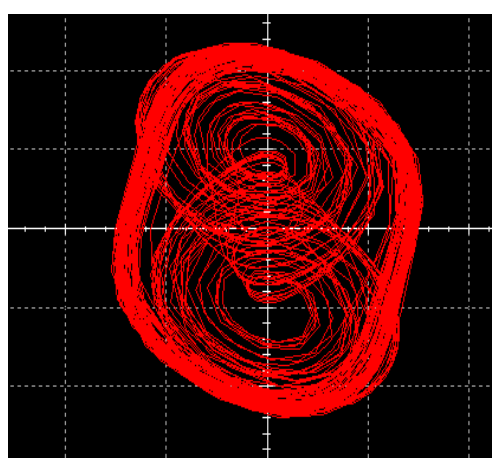

(d)

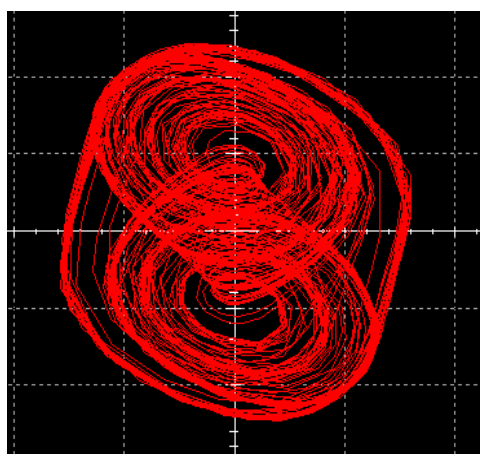

(f)

Fig. 11 Coexistence of transient attractors with different chaotic times observed from Multisim. Where (a), (c), and (e) are the time domain waveforms in the $\mathrm{v}_{\mathrm{C} 2}$ plane, and (b), (d) and (f) are the phase portraits in the $\mathrm{v}_{\mathrm{C} 1}-\mathrm{v}_{\mathrm{C} 2}$ plane; (a) and (b) with $v_{C 40}=0.7 v$, (c) and (d) with $v_{C 40}=0.043 v$, (e) and (f) with $v_{C 40}=-0.5 v$.

Supposing the time constant $R C=10 \mu \mathrm{s}$, if $R=10 \mathrm{k} \Omega$, the capacitances are configured as $C_{1}=C_{2}=C_{3}=C_{4}=C=1 n F$. Based on the synaptic connection weight matrix defined in Eq. (3), some 
resistances can be deduced as $R_{1}=R / 1.5=6.667 \mathrm{k} \Omega, \quad R_{2}=R / 2=5 \mathrm{k} \Omega, \quad R_{3}=R / 0.9=11.111 \mathrm{k} \Omega$, $R_{4}=R / 1.5=6.667 \mathrm{k} \Omega, \quad R_{5}=R / 1.5=6.667 \mathrm{k} \Omega, \quad R_{8}=R / 3=3.333 k \Omega \quad, \quad R_{9}=R / 2=5 \mathrm{k} \Omega \quad, \quad$ and $R_{10}=R / 0.8=12.5 \mathrm{k} \Omega$. In addition to these resistances, other resistances are relevant with the system parameters. In other words, when the representative parameters $a=1.5, b=-0.05, k_{1}=-0.3, k_{2}=-0.1$ are chosen and the gain of the amplifiers are both set as $g=0.1$, we can determine that $R_{6}=R /\left|a k_{1}\right|=22.222 k \Omega, \quad R_{7}=g^{2} R /\left|3 b k_{1}\right|=2.222 k \Omega, \quad R_{11}=R /\left|k_{2}\right|=100 \mathrm{k} \Omega$.

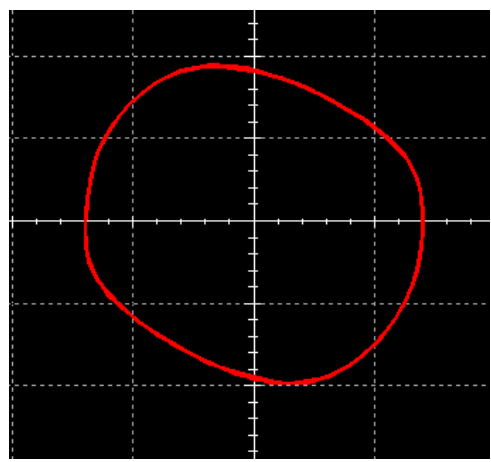

(a)

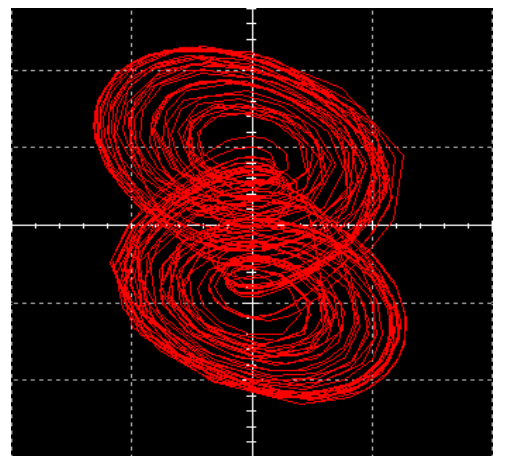

(c)

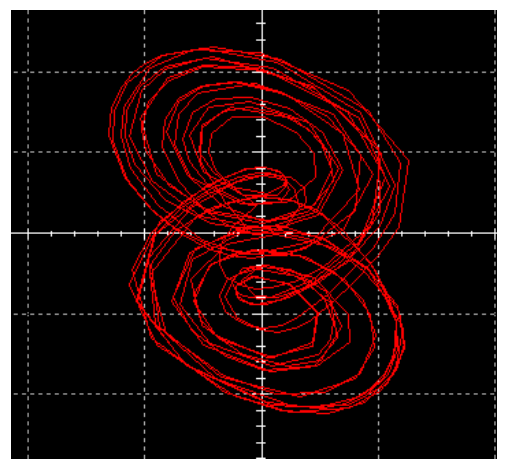

(b)

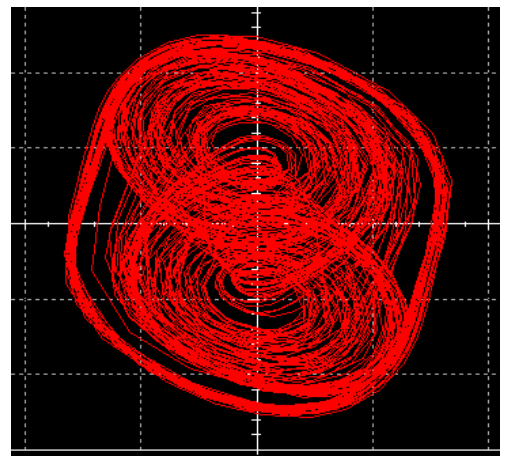

(d)

Fig. 12 Captured attractors with different capacitor voltages of $C_{1}-C_{4}$ in the $\mathrm{v}_{\mathrm{Cl}}-\mathrm{v}_{\mathrm{C} 2}$ plane, (a) periodic attractor for $(-0.1 v,-0.1 v, 0.1 v, 0.1 v)$; (b) quasi-periodic attractor for $(0.05 v, 0 v, 0 v, 0.4 v)$; (c) chaotic attractor for $(-0.1 v,-0.1 v,-0.1 v, 0 v)$; (d) transient chaotic attractor for $(-0.1 v,-0.1 v,-0.1 v,-0.1 v)$.

Based on the circuit schemes given in Fig.9 and Fig. 10, an analogue circuit is built up in Multisim, where the bipolar NPN transistors MPS2222, operational amplifiers TL082CP and multipliers AD633JN supplied by $\pm 15 \mathrm{~V}$ DC voltage, resistors and capacitors are employed as the discrete components. Furthermore, two external DC current sources are added to the inverting input of the operational amplifiers $\mathrm{U}_{1}$ and $\mathrm{U}_{3}$ respectively as the dual bias currents $I$. In Multisim simulation, the initial condition of the neuron can be easily realized by adjusting the initial voltage across the capacitor, and the phase portrait and time domain waveform can be also observed conveniently. When the DC current sources are set as $-0.0001 \mathrm{~mA}$ and the maximum simulation time step is chosen as $10^{-5}$, the Multisim simulations can be 
obtained. First, with the initial capacitor voltages of $C_{1}, C_{2}$ and $C_{3}$ are fixed as $(0.1 v, 0 v, 0.1 v)$, but adjusting the initial voltage of $C_{4}$, the screen shots of the time domain waveforms in the $v_{C 2}$ plane and the phase portraits in the $v_{C 1}-v_{C 2}$ plane are displayed in Fig. 11. As can be seen, the circuit simulations match with the theoretical results in Fig. 4 basically.

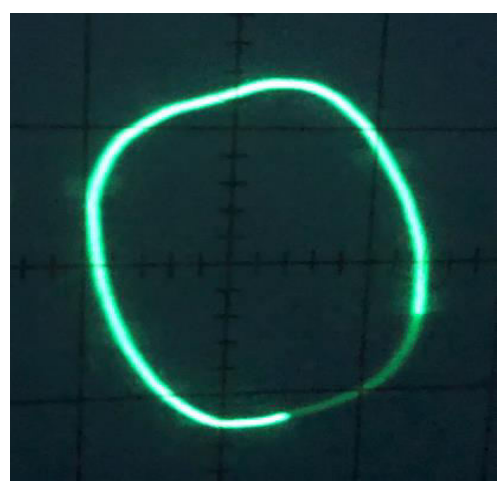

(a)

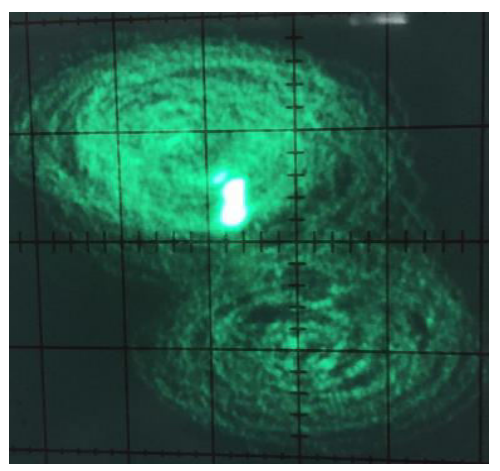

(c)

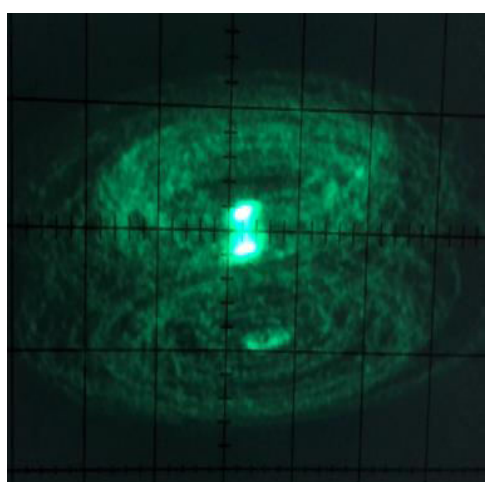

(b)

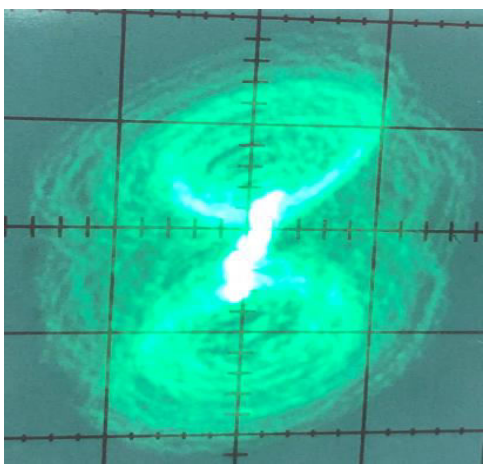

(d)

Fig. 13 Hardware experimental measurements in the $\mathrm{v}_{\mathrm{C} 1}-\mathrm{v}_{\mathrm{C} 2}$ plane: (a) periodic attractor; (b) quasi-periodic attractor; (c) chaotic attractor; (d) transient chaotic attractor.

In the same manner, the coexisting four attractors in the $v_{C 1}-v_{C 2}$ plane under different initial voltages of the four capacitors $C_{1}, C_{2}, C_{3}$ and $C_{4}$ can be also measured as shown in Fig. 12, and Fig. 12 is in good agreement with Fig. 8. Similarly, the experimental circuit is made manually on breadboard and the results are captured by an oscilloscope in X-Y mode as shown in Fig. 13. Ignoring some minor deviations that caused by the idealization of mathematical model and the existence of parasitic parameters in circuit, Fig. 13 are almost the same as Fig.8 and Fig. 12.

\section{Conclusion}

In this paper, by adding electromagnetic radiation on the second neuron and imposing the same magnitude of dual bias currents to the first neuron and third neuron, a small neural network with three neurons is studied systematically. Through bifurcation diagram, Lyapunov exponent spectrum, phase 
portrait and time domain waveform, we find that with suitable system parameters and initial conditions, many types of attractors and parallel bifurcation phenomenon are emerged in this neural network. Notably that under different initial magnetic flux, which is the magnetic flux across the inner and outer cell membrane of the second neuron affected by the electromagnetic radiation, there are successive transient chaotic attractors with different chaotic times in this model, which indicates the multistability characteristic of the system. Finally, Multisim simulations and hardware experiments are carried out to verify the validity of the numerical analyses. These distinctive results may provide a meaningful reference for human brain activities on our future research.

\section{Acknowledgement}

The authors would like to thank the project supported by the National Natural Science Foundation of China (Grant No. 61901169) and the Natural Science Foundation of Hunan Province, China (Grant No. 2019JJ40190).

\section{Data availability statement}

The datasets generated and/or analyzed during the current study are available from the corresponding author on a reasonable request.

\section{Declarations}

\section{Conflict of interest}

The authors declare that they have no conflict of interest.

\section{References}

[1]. Luke Volgin, Duncan Taylor, Jo-Anne Bright, Meng-Han Lin. Validation of a neural network approach for STR typing to replace human reading. Forensic Science International: Genetics 2021; 55(11): 102951.

[2]. Ying Xu, Jun Ma, Xuan Zhan, Lijian Yang, Ya Jia. Temperature effect on memristive ion channels. Cognitive Neurodynamics 2019; 13(6): 601-611.

[3]. Chunsheng Li, Shiyue Liu, Zeyu Wang. Classifying Interictal Epileptiform Activities in Intracranial EEG Using Complex-Valued Convolutional Neural Network. International Journal of Psychophysiology 2021; 168(S): S104-S105. 
[4]. Leon O. Chua. Memristor-The Missing Circuit Element. IEEE Transactions on Circuit Theory 1971; 18(5): 507-519.

[5]. Qiuzhen Wan, Zhaoteng Zhou, Wenkui Ji, Fei Yu, Chunhua Wang. Dynamic Analysis and Circuit Realization of a Novel No-Equilibrium 5D Memristive Hyperchaotic System with Hidden Extreme Multistability. Complexity 2020; Article ID 7106861.

[6]. Qingdu Li, Song Tang, Hongzheng Zeng, Tingting Zhou. On hyperchaos in a small memristive neural network. Nonlinear Dynamics 2014; 78(2): 1087-1099.

[7]. Chengjie Chen, Jingqi Chen, Han Bao, Mo Chen, Bocheng Bao. Coexisting multi-stable patterns in memristor synapse-coupled Hopfield neural network with two neurons. Nonlinear Dynamics 2019; 95(4): 3385-3399.

[8]. Zhiri Tang, Yanhua Chen, Zhihua Wang, Ruihan Hu, Edmond Q. Wu. Non-spike timing-dependent plasticity learning mechanism for memristive neural networks. Applied Intelligence 2021; 51(1): 1-12.

[9]. Chunbo Xiu, Ruxia Zhou, Yuxia Liu. New chaotic memristive cellular neural network and its application in secure communication system. Chaos, Solitons \& Fractals 2020; 141(12): 110316.

[10]. Wei Zhang, Jiangtao Qi. Synchronization of coupled memristive inertial delayed neural networks with impulse and intermittent control. Neural Computing and Applications 2020; 33(6): 1-12.

[11]. Yanyi Cao, Wenjun Jiang, Jiahai Wang. Anti-synchronization of delayed memristive neural networks with leakage term and reaction-diffusion terms. Knowledge-Based Systems 2021; 233(12): 107539.

[12]. Yuanyuan Liu, Zhongkui Sun, Xiaoli Yang, Xu Wei. Dynamical robustness and firing modes in multilayer memristive neural networks of nonidentical neurons. Applied Mathematics and Computation 2021; 409(11): 126384.

[13]. Hairong Lin, Chunhua Wang, Quanli Deng, Cong Xu, Zekun Deng, Chao Zhou. Review on chaotic dynamics of memristive neuron and neural network. Nonlinear Dynamics 2021; 106(1): 959-973.

[14]. J. J. Hopfield. Neurons with Graded Response Have Collective Computational Properties like Those of Two-State Neurons. PNAS 1984; 81(10): 3088-3092.

[15]. Leng Yang, Dongsheng Yu, Yihua Hu, Samson Shenglong Yu, Zongbin Ye. Dynamic behaviors of hyperbolic-type memristor-based Hopfield neural network considering synaptic crosstalk. Chaos 2020; 30(3): 033108.

[16]. Z. T. Njitacke, J. Kengne, H. B. Fotsin. A plethora of behaviors in a memristor based Hopfield neural networks (HNNs). International Journal of Dynamics \& Control 2019; 7(1): 36-52.

[17]. Z. T. Njitacke, J. Kengne, H. B. Fotsin. Coexistence of Multiple Stable States and Bursting Oscillations in a 4D Hopfield Neural Network. Circuits, Systems, and Signal Processing, 2020; 39(7):3424-3444.

[18]. Bocheng Bao, Hui Qian, Jiang Wang, Quan Xu, Mo Chen, Huagan Wu, Yajuan Yu. Numerical analyses and experimental validations of coexisting multiple attractors in Hopfield neural network. Nonlinear Dynamics 2017; 90(4): 2359-2369.

[19]. Paulo C. Rech. Chaos and hyperchaos in a Hopfield neural network. Neurocomputing 2011; 74(17): 3361-3364.

[20]. Marius-F. Danca, Nikolay Kuznetsov. Hidden chaotic sets in a Hopfield neural system. Chaos Solitons \& Fractals 2017; 103(10): 144-150. 
[21]. Bocheng Bao, Chengjie Chen, Han Bao, Xi Zhang, Quan Xu, Mo Chen. Dynamical Effects of Neuron Activation Gradient on Hopfield Neural Network: Numerical Analyses and Hardware Experiments. International Journal of Bifurcation and Chaos 2019; 29(4): 1930010.

[22]. Isaac S D, Z. T. Njitacke, J. Kengne. Effects of Low and High Neuron Activation Gradients on the Dynamics of a Simple 3D Hopfield Neural Network. International Journal of Bifurcation and Chaos 2020; 30(11): 2050159.

[23]. Hu Wang, Yongguan Yu, Guoguang Wen, Shuo Zhang, Junzhi Yu. Global stability analysis of fractional-order Hopfield neural networks with time delay. Neurocomputing 2015; 154(4): 15-23.

[24]. Chaouki Aouiti, Foued Miaadi. Pullback attractor for neutral Hopfield neural networks with time delay in the leakage term and mixed time delays. Neural Computing and Applications 2019; 31(8): 4113-4122.

[25]. Xiaoyu Hu, Chongxin Liu, Ling Liu, Junkang Ni, Yapeng Yao. Chaotic dynamics in a neural network under electromagnetic radiation. Nonlinear Dynamics 2018; 91(3): 1541-1554.

[26]. Kwan Patrick, Brodie Martin J. Early Identification of Refractory Epilepsy. The New England Journal of Medicine 2000; 342(5): 314-319.

[27]. Shirin Panahi, Zainab Aram, Sajad Jafari, Jun Ma. Modeling of epilepsy based on chaotic artificial neural network. Chaos, Solitons \& Fractals 2017; 105(12): 150-156.

[28]. Hairong Lin, Chunhua Wang. Influences of electromagnetic radiation distribution on chaotic dynamics of a neural network. Applied Mathematics and Computation, 2020, 369(3): 124840.

[29]. Reuven Sandyk, P. A. Anninos, N. Tsagas, K. Derpapas. Magnetic Fields in the Treatment of Parkinson's Disease. International Journal of Neuroscience 1992; 63(1-2): 141-150.

[30]. Reuven Sandyk. Alzheimer's Disease: Improvement of Visual Memory and Visuoconstructive Performance by Treatment with Picotesla Range Magnetic Fields. International Journal of Neuroscience 1994; 76(3-4): 185-225.

[31]. F. M. Allehiany, Emad E. Mahmoud, Lone Seth Jahanzaib, Pushali Trikha, Hammad Alotaibi. Chaos Control and Analysis of Fractional Order Neural Network under Electromagnetic Radiation. Results in Physics 2021; 21(11): 103786.

[32]. Yu Fei, Zinan Zhang, Hui Shen, Yuanyuan Huang, Shuo Cai, Jie Jin, Sichun Du . Design and FPGA Implementation of a Pseudo-random Number Generator Based on a Hopfield Neural Network under Electromagnetic Radiation. Frontiers in Physics 2021; 9(6): 690651.

[33]. Toomey Emily, Segall Ken, Berggren Karl K. Design of a Power Efficient Artificial Neuron Using Superconducting Nanowires. Frontiers in neuroscience 2019; 13(9): 933.

[34]. Wei-Mien M. Hsu, David B. Kastner, Stephen A. Baccus, Tatyana O. Sharpee. How inhibitory neurons increase information transmission under threshold modulation. Cell Reports 2021; 35(8): 109158.

[35]. Hassan Saberi Nik, Sohrab Effati, JAFAR Saberi-Nadjafi. Ultimate bound sets of a hyperchaotic system and its application in chaos synchronization. Complexity 2015; 20(4): 30-44.

[36]. Bao Bocheng, Hui Qian, Quan Xu, Mo Chen, Jiang Wang, Yajuan Yu. Coexisting Behaviors of Asymmetric Attractors in Hyperbolic-Type Memristor based Hopfield Neural Network. Frontiers in computational neuroscience 2017; 11(8): 1-14. 
[37]. Hairong Lin, Chunhua Wang, Yumei Tan. Hidden extreme multistability with hyperchaos and transient chaos in a Hopfield neural network affected by electromagnetic radiation. Nonlinear Dynamics 2020; 99(4): 2369-2386.

[38]. Sayantan Nag Chowdhury, Dibakar Ghosh. Hidden attractors: A new chaotic system without equilibria. The European Physical Journal Special Topics 2020; 229(6-7): 1299-1308.

[39]. Tél Tamás. The joy of transient chaos. Chaos 2015; 25(9): 097619.

[40]. V. Kamdoum Tamba, E. R. Feudjio, F. Kapche Tagne, J. Noumbissie Fankam, H. B. Fotsin. Crisis event, hysteretic dynamics inducing coexistence of attractors and transient chaos in an autonomous RC hyperjerk like-chaotic circuit with cubic nonlinearity. The European Physical Journal Special Topics 2020; 229(6-7): 1189-1210.

[41]. Z. T. Njitacke, J. Kengne, T. Fonzin Fozin, B. P. Leutcha, H. B. Fotsin. Dynamical analysis of a novel 4-neurons based Hopfield neural network: emergences of antimonotonicity and coexistence of multiple stable states. International Journal of Dynamics and Control 2019; 7(3): 823-841.

[42]. Z. T. Njitacke, Sami Doubla Isaac, J. Kengne, A. Nguomkam Negou, Gervais Dolvis Leutcho. Extremely rich dynamics from hyperchaotic Hopfield neural network: Hysteretic dynamics, parallel bifurcation branches, coexistence of multiple stable states and its analog circuit implementation. The European Physical Journal Special Topics 2020; 229(6-7): 1133-1154.

[43]. J. Kengne, S. M. Njikam, V. R. Folifack Signing. A plethora of coexisting strange attractors in a simple jerk system with hyperbolic tangent nonlinearity. Chaos, Solitons and Fractals 2018; 106(1): 201-213.

[44]. Fuqiang Wu, Jun Ma, Ge Zhang. A new neuron model under electromagnetic field. Applied Mathematics and Computation 2019; 347(4): 590-599.

[45]. Shukai Duan, Xiaofeng Liao. An electronic implementation for Liao's chaotic delayed neuron model with non-monotonous activation function. Physics Letters A 2007; 369(1-2): 37-43.

[46]. Shukai Duan, Lidan Wang. A novel delayed chaotic neural model and its circuitry implementation. Computers and Mathematics with Applications 2008; 57(11-12): 1736-1742.

[47]. Quan Xu, Zhe Song, Hui Qian, Mo Chen, Pingye Wu, Bocheng Bao. Numerical analyses and breadboard experiments of twin attractors in two-neuron-based non-autonomous Hopfield neural network. The European Physical Journal Special Topics 2018; 227(7-9): 777-786.

[48]. Quan Xu, Zhe Song, Han Bao, Mo Chen, Bocheng Bao. Two-neuron-based non-autonomous memristive Hopfield neural network: Numerical analyses and hardware experiments. AEUE - International Journal of Electronics and Communications 2018; 96(11): 66-74. 